\title{
CQ-free optimality conditions and strong dual formulations for a special conic optimization problem
}

\author{
Olga Kostyukova ${ }^{1}$, Tatiana Tchemisova ${ }^{2, *}$ \\ ${ }^{1}$ Institute of Mathematics, National Academy of Sciences of Belarus, Minsk, Belarus \\ ${ }^{2}$ Center for Research and Development in Mathematics and Applications, University of Aveiro, Aveiro, Portugal
}

\begin{abstract}
In this paper, we consider a special class of conic optimization problems, consisting of set-semidefinite (or $K$ semidefinite) programming problems, where the set $K$ is a polyhedral convex cone. For these problems, we introduce the concept of immobile indices and study the properties of the set of normalized immobile indices and the feasible set. This study provides the main result of the paper, which is to formulate and prove the new first-order optimality conditions in the form of a criterion. The optimality conditions are explicit and do not use any constraint qualifications. For the case of a linear cost function, we reformulate the $K$-semidefinite problem in a regularized form and construct its dual. We show that the pair of the primal and dual regularized problems satisfies the strong duality relation which means that the duality gap is vanishing.
\end{abstract}

Keywords Conic optimization, set-semidefinite optimization, optimality conditions, normalized immobile index set, regularized dual problem, strong duality

AMS 2010 subject classifications 90C25, 90C30, 90C34

DOI: $10.19139 /$ soic-2310-5070-915

\section{Introduction}

Convex optimization is a subfield of mathematical optimization where the problems of minimizing convex functions over convex sets are solved. Convex optimization has applications in a wide range of disciplines, such as automatic control systems, estimation and signal processing, communications and networks, electronic circuit design, data analysis and modeling, statistics, optimal experimental design, and structural optimization, where the approximation concept has proven to be efficient ( see [7,8] and the references therein). With recent advancements in computing and optimization algorithms, convex programming is indispensable in emerging methods of data mining, analytics, machine learning, as well as management, economics, and finance (e.g.[23, 27, 31, 32]).

Conic optimization studies problems of minimizing a convex function over the intersection of an affine subspace and a convex cone. Conic models are efficiently used in dynamic processes, biomedical and chemical engineering, biology, credit risk optimization, etc. (see [3, 32, 35], and others). According to F. Glineur ([13]), conic optimization is an elegant framework for convex optimization, due to the fact that conic formulations present various advantages over the traditional formulations: the standard primal-dual pair of conic problems does feature a great deal of symmetry, the dual problem and the associated weak and strong duality properties can be derived in a "seamless manner" once the dual cone has been computed, and the conic formulations are helpful when designing and analyzing numerical algorithms.

\footnotetext{
*Correspondence to: Tatiana Tchemisova (Email: tatiana@ua.pt). Center for Research and Development in Mathematics and Applications, Department of Mathematics, University of Aveiro, Campus Universitário Santiago, 3810-193, Aveiro, Portugal.
}

ISSN 2310-5070 (online) ISSN 2311-004X (print)

Copyright (C) 2020 International Academic Press 
In this paper, we consider a special class of conic optimization problems, consisting of set-semidefinite (or $K$ semidefinite) programming problems, where the set $K$ is a polyhedral convex cone. The interest in such problems is due to the fact that they have a wide range of applications in such important areas as systems and management theory, robust and combinatorial optimization. Among the most important subclasses of conic problems under consideration, there are semidefinite programming (SDP) and copositive problems which have many applications in robust optimization, graph theory, computational biology, systems and control theory, sensor network location, and data analysis, among others [2, 9, 22, 24]. Semidefinite and copositive programming are recognized in combinatorial optimization as valuable techniques for modeling and obtaining bounds on solutions of $\mathcal{N} \mathcal{P}$ - hard problems (for the references see [5, 10, 14, 33]). New numerical scheme based on semidefinite programming to compute approximately and sometimes exactly the Nash equilibria, the min-max of any finite game, and the values and the optimal strategies of any polynomial 2-player zero-sum game are presented in [21,34] et. al.

One of the main difficulties faced in solving conic optimization problems is that many of the problems in this class are characterized by a violation of regularity (and/or so-called $C Q-$ constraint qualifications). This makes it impossible to apply the known results from the general theory of optimization, entails a violation of strict duality relations which in turn leads to significant difficulties in justifying and implementing numerical methods for solving such problems. In this regard, it is relevant to study the problems of conic optimization without additional assumptions about regularity.

The purpose of this work is to formulate and prove new optimality conditions which do not use any CQs (are $C Q$-free) and obtain new dual formulations which satisfy the strong duality relations and therefore may form the basis of efficient numerical methods.

The paper is organized as follows. Section 1 hosts Introduction. The problem's settings and basic notations are introduced in section 2. The new optimality conditions for a set-semidefinite problem with a polyhedral set are formulated in section 3. In section 4, we explore the properties of the set of immobile indices and the feasible set of the problem under study and use them to prove, in section 5, the main result of the paper, which is the new first-order CQ-free optimality conditions for the set-semidefinite programming (Theorem 1). In section 6, for a linear case, we deduce a regularized pair of primal and dual $K$-semidefinite problems and show that for this pair the strong duality property holds. The final section 7 contains some conclusions.

\section{Problem's settings and basic notation}

In what follows, we will use the following notation. Given integers $p$ and $k$, let $\mathbb{R}^{p}$ and $\mathbb{R}^{k \times p}$ denote the set of all real-valued $p$ - vectors and $k \times p$ - matrices, respectively. Denote by $\mathbb{R}_{+}^{p} \subset \mathbb{R}^{p}$ the subset of all $p$ - vectors with non-negative components and by $\mathcal{S}(p)$ the space of symmetric $p \times p$ matrices with the trace inner product:

$$
A \bullet B:=\operatorname{trace}(A B), \text { for } A, B \in \mathcal{S}(p) .
$$

For an arbitrary nonempty set $K \subset \mathbb{R}^{p}$, consider the set

$$
\mathcal{C}^{K}:=\left\{\mathcal{D} \in \mathcal{S}(p): t^{\top} \mathcal{D} t \geq 0 \quad \forall t \in K\right\}
$$

called a cone of $K$ - semidefinite (set-semidefinite) matrices, or simply, the $K$ - semidefinite cone, see e.g. [11, 12]. It worth mentioning that the cone $\mathcal{C}^{K}$ is a generalization of a well-known and well-studied in literature cone of positive semidefinite matrices

$$
\mathcal{S}_{+}(p):=\left\{\mathcal{D} \in S(p): t^{\top} \mathcal{D} t \geq 0 \forall t \in \mathbb{R}^{p}\right\},
$$

and a less studied cone of copositive matrices

$$
\mathcal{C O P}(p):=\left\{\mathcal{D} \in S(p): t^{\top} \mathcal{D} t \geq 0 \forall t \in \mathbb{R}_{+}^{p}\right\},
$$

see e.g. $[1,4,5,6]$ and the references therein. Indeed, for $K=\mathbb{R}^{p}$, we have $\mathcal{C}^{K}=\mathcal{S}_{+}(p)$ and for $K=\mathbb{R}_{+}^{p}$, we have $\mathcal{C}^{K}=\mathcal{C O P}(p)$ 
Some properties of the cone of $K$-semidefinite matrices are studied in [12]. Notice, in particular, that this cone is convex for any set $K$.

Let $\mathcal{A}: \mathbb{R}^{n} \rightarrow \mathcal{S}(p)$ and $c: \mathbb{R}^{n} \rightarrow \mathbb{R}$ be some given continuous maps. A problem

$$
\text { minimize } c(x), \quad \text { subject to } x \in \mathbb{R}^{n}, \mathcal{A}(x) \in \mathcal{C}^{K},
$$

is called a set-semidefinite (or $\mathrm{K}$-semidefinite) optimization problem. This problem can be considered as a particular case of conic optimization problems.

In this paper, we consider problem (2) under assumptions that the map $\mathcal{A}: \mathbb{R}^{n} \rightarrow \mathcal{S}(p)$ is affine, the function $c: \mathbb{R}^{n} \rightarrow \mathbb{R}$ is convex and the set $K \subset \mathbb{R}^{p}$ is a polyhedral convex cone. Namely, we consider the following optimization problem:

$$
\begin{gathered}
\text { minimize } c(x), \\
\text { subject to } x \in \mathbb{R}^{n}, t^{\top} \mathcal{A}(x) t \geq 0 \quad \forall t \in K:=\left\{t \in \mathbb{R}^{p}: \mathbf{A} t=0, \mathbf{B} t \geq 0\right\},
\end{gathered}
$$

where $\mathcal{A}(x):=\sum_{k=1}^{n} A_{k} x_{k}+A_{0}, A_{k} \in \mathcal{S}(p), k=0,1, \ldots, n, \mathbf{A} \in \mathbb{R}^{m_{0} \times p}, \mathbf{B} \in \mathbb{R}^{m_{*} \times p}$, are given matrices.

Problem (3) is a generalization of semidefinite and copositive programming problems since in the case $K=\mathbb{R}^{p}$, it is a problem of SDP, and in the case $K=\mathbb{R}_{+}^{p}$, a copositive problem.

The main purpose of this paper is to prove the optimality conditions and to obtain strong duality results for the set-semidefinite problem (3) that do not require any regularity conditions (constraint qualifications or, shortly, CQ). We will use here the approach developed in our previous papers (see e.g. $[16,18,20]$ ) for different classes of convex problems.

\subsection{Some other formulations}

It is evident that problem (3) can be considered as a special case of Semi-Infinite Programming (SIP) problems [7]. On the other hand, some more general than (3) problems can be reduced to it. In fact, consider a SIP problem in the form

$$
\text { minimize } c(x), \quad \text { subject to } x \in \mathbb{R}^{n}, f(x, t) \geq 0 \quad \forall t \in M,
$$

where the decision variable $x=\left(x_{1}, \ldots, x_{n}\right)$ is an $n$-vector, the index variable $t=\left(t_{1}, \ldots, t_{p}\right)$ is a $p$-vector, and $M \subset \mathbb{R}^{p}$ is a given index set.

Suppose that in (4), the constraint function $f(x, t)$ is linear w.r.t. $x$ and linear- quadratic w.r.t. $t$, the cost function $c(x), x \in \mathbb{R}^{n}$, is convex, and the index set $M$ is a convex polyhedron:

$$
M:=\left\{t \in \mathbb{R}^{p}: \overline{\mathbf{A}} t=\mathbf{a}, \overline{\mathbf{B}} t \geq \mathbf{b}\right\},
$$

with given matrices $\overline{\mathbf{A}} \in \mathbb{R}^{\bar{m}_{0} \times p}, \overline{\mathbf{B}} \in \mathbb{R}^{\bar{m}_{*} \times p}$ and vectors $\mathbf{a} \in \mathbb{R}^{\bar{m}_{0}}, \mathbf{b} \in \mathbb{R}^{\bar{m}_{*}}$.

Problem (4) with the index set in the form (5) is a special case of polinomial SIP problems.

Let us show that problem (4) with the index set in the form (5) and with the constraints function $f(x, t)$ described above, can be reduced to problem (3).

Indeed, it is evident that problem (4) can be rewritten as follows:

$$
\begin{gathered}
\text { minimize } c(x), \\
\text { subject to } x \in \mathbb{R}^{n}, t^{\top} \overline{\mathcal{A}}(x) t+2 t^{\top}\left(D x+d_{0}\right)+q^{\top} x+q_{0} \geq 0 \forall t \in M,
\end{gathered}
$$

where the matrix function $\overline{\mathcal{A}}(x)$ has the form $\overline{\mathcal{A}}(x):=\sum_{k=1}^{n} \bar{A}_{k} x_{k}+\bar{A}_{0}$, and the problem's data is set: matrices $\bar{A}_{k} \in \mathcal{S}(p), k=0,1, \ldots, n, D \in \mathbb{R}^{p \times n}$, vectors $d_{0} \in \mathbb{R}^{p}, q \in \mathbb{R}^{n}$, and the number $q_{0} \in \mathbb{R}$. 
Let $X_{1}$ be the set of feasible solutions of problem (6):

$$
X_{1}=\left\{x \in \mathbb{R}^{n}: t^{\top} \overline{\mathcal{A}}(x) t+2 t^{\top}\left(D x+d_{0}\right)+q^{\top} x+q_{0} \geq 0 \quad \forall t \in M\right\} .
$$

Denote by $d_{. k} \in \mathbb{R}^{p}, k=1, \ldots, n$, the columns of the matrix $D \in \mathbb{R}^{p \times n}: D=\left(d_{\cdot 1} \ldots d_{. n}\right)$, and let $q=\left(q_{1}, \ldots, q_{n}\right)^{\top}$. Introduce the following notation:

$$
\begin{aligned}
& B_{k}:=\left(\begin{array}{cc}
q_{k} & d_{\dot{A}_{k}}^{\top} \\
d_{\cdot k} & \bar{A}_{k}
\end{array}\right) \in \mathcal{S}(p+1), k=0,1, \ldots, n ; \mathcal{B}(x):=\sum_{k=1}^{n} B_{k} x_{k}+B_{0}, \\
& \bar{\tau}:=\left(\tau_{0}, \tau^{\top}\right)^{\top}, \text { where } \tau_{0} \in \mathbb{R}, \tau=\left(\tau_{1}, \ldots, \tau_{p}\right)^{\top} \in \mathbb{R}^{p} \\
& \bar{K}:=\left\{\bar{\tau} \in \mathbb{R}^{p+1}:(-\mathbf{a}, \overline{\mathbf{A}}) \bar{\tau}=0,(-\mathbf{b}, \overline{\mathbf{B}}) \bar{\tau} \geq 0, \tau_{0} \geq 0\right\}
\end{aligned}
$$

and consider the set

$$
X_{2}:=\left\{x \in \mathbb{R}^{n}: \bar{\tau}^{\top} \mathcal{B}(x) \bar{\tau} \geq 0 \forall \bar{\tau} \in \bar{K}\right\} .
$$

One can easily prove the following lemma.

\section{Lemma 1}

Given the feasible set $X_{1}$ of problem (6) and the set $X_{2}$ defined in (7), the equality $X_{1}=X_{2}$ holds.

Note that the set $X_{2}$ can be considered as the feasible set of the problem in the form (3) with function $\mathcal{A}(x)$ replaced by $\mathcal{B}(x)$ and the index set $K$ substituted by $\bar{K}$.

It follows from Lemma 1, that the study of the special SIP problem in the form (6) with the index set (5) is equivalent to the study of the conic (set-semidefinite) problem in the form (3).

\subsection{Normalized set of immobile indices}

In accordance with the generally accepted definition (see, e.g., [7, 13]), problem (3) satisfies the Slater condition if for some $\bar{x} \in \mathbb{R}^{n}$ it holds: $t^{\top} \mathcal{A}(\bar{x}) t>0 \quad \forall t \in K \backslash\{0\}$. Notice that from the very beginning, we do not make assumptions that the constraints of problem (3) satisfy the Slater condition or some other conditions.

Since the set $K$ is a cone, problem (3) has an unbounded index set. It is easy to show that this problem is equivalent to the following SIP problem with a compact index set:

$$
\begin{gathered}
\text { minimize } c(x), \\
\text { subject to } x \in \mathbb{R}^{n}, t^{\top} \mathcal{A}(x) t \geq 0 \quad \forall t \in T:=\left\{t \in K:\|t\|_{\infty}=1\right\},
\end{gathered}
$$

where $\|t\|_{\infty}=\max _{k \in P}\left|t_{k}\right|$. The index set $T$ is normalized in this problem.

In our papers $[15,16,19]$, and others, we introduced for convex SIP problems the concept of immobile indices (the indices of constraints which are active for all feasible solutions) and showed that these indices play a special role in formulating the optimality conditions which do not need the fulfillment of the Slater condition. Here, we will show how our approach can be applied to the conic problem under consideration. (3):

Denote by $X$ the set of feasible solutions in the SIP problem (8) and in the equivalent $K$ - semidefinite problem

$$
X=\left\{x \in \mathbb{R}^{n}: t^{\top} \mathcal{A}(x) t \geq 0 \quad \forall t \in T\right\},
$$

and by $T_{i m}$ the set of (normalized) immobile indices in problem (8):

$$
T_{i m}:=\left\{t \in T: t^{\top} \mathcal{A}(x) t=0 \quad \forall x \in X\right\} .
$$

It can be proved (see [15]) that the Slater condition for problem (8) is equivalent to the condition $T_{i m}=\emptyset$. 
It will be shown (see Lemma 3 below) that the set $T_{i m}$ is either empty or a union of a finite number of convex bounded polyhedra. Then, evidently, the set conv $T_{i m}$ is either empty or a convex polyhedron. Here conv $\mathcal{D}$ stays for the convex hull of a set $\mathcal{D}$.

In what follows, we will use the vertices of the set conv $T_{i m}$. Denote the set of these vertices by

$$
\left\{t^{*}(j) \in T_{i m}, j \in J,|J|<\infty\right\} .
$$

In the case $T_{i m}=\emptyset$, we set $J=\emptyset$.

\section{CQ-free optimality criteria}

The main aim of this paper is to prove necessary and sufficient optimality conditions for the conic optimization problem (3) and the equivalent problem (8). In this section, we formulate two theorems which have the form of criteria. These theorems do not demand that the feasible set of the problem satisfies any constraints qualifications and therefore, we refer to them as to $C Q$-free optimality conditions. The main concepts that allow us to formulate and prove these optimality conditions are the set of immobile indices and the vertices of its convex hull.

\section{Theorem 1}

A vector $x^{0} \in X$ is an optimal solution of the set-semidefinite problem (3) (and the equivalent SIP problem (8)) iff there exist vectors

$$
\lambda(j) \in K, j \in J ; \tau(i) \in K, i \in I,|I| \leq n,
$$

such that

$$
(U+V) \bullet A_{k}=\frac{\partial c\left(x^{0}\right)}{\partial x_{k}}, k=1, \ldots, n ; \quad(U+V) \bullet \mathcal{A}\left(x^{0}\right)=0,
$$

where matrices $U$ and $V$ are defined as follows:

$$
U:=\sum_{i \in I} \tau(i)(\tau(i))^{\top}, \quad V:=\sum_{j \in J}\left(\lambda(j)\left(t^{*}(j)\right)^{\top}+t^{*}(j)(\lambda(j))^{\top}\right),
$$

and $t^{*}(j), j \in J$, are vertices of the polyhedron conv $T_{i m}$.

We will prove this theorem in section 5 .

For applications, it may be useful to reformulate Theorem 1 in a different way.

Set $\bar{m}:=|J|$ and denote by $\mathbb{R}^{p \times \bar{m}}(K)$ the set of $p \times \bar{m}$ matrices $\Lambda=(\lambda(j), j \in J)$ with $\lambda(j) \in K, j \in J$.

Having introduced the matrix $\mathcal{T}:=\left(t^{*}(j), j \in J\right) \in \mathbb{R}^{p \times \bar{m}}$ and the sets of matrices

$$
\begin{gathered}
\mathcal{V}(K):=\left\{V \in \mathcal{S}(p): V=\Lambda \mathcal{T}^{\top}+\mathcal{T} \Lambda^{\top}, \Lambda \in \mathbb{R}^{p \times \bar{m}}(K)\right\}, \\
\left(\mathcal{C}^{K}\right)^{*}:=\operatorname{conv}\left\{t t^{\top}: t \in K\right\}
\end{gathered}
$$

we can reformulate Theorem 1 as follows.

\section{Theorem 2}

A vector $x^{0} \in X$ is an optimal solution of problem (3) iff there exist matrices $U^{0} \in\left(\mathcal{C}^{K}\right)^{*}$ and $V^{0} \in \mathcal{V}(K)$ such that

$$
\left(U^{0}+V^{0}\right) \bullet A_{k}=\frac{\partial c\left(x^{0}\right)}{\partial x_{k}}, k=1,2, \ldots, n ; \quad\left(U^{0}+V^{0}\right) \bullet \mathcal{A}\left(x^{0}\right)=0 .
$$

At the end of this section, we would like to make several remarks.

Remark 1

It can be shown that the cone $\left(\mathcal{C}^{K}\right)^{*}$ is dual to the cone $\mathcal{C}^{K}$. 


\section{Remark 2}

Theorem 1 can be easily generalized for the case when the set $K$ is an union of the finite number of polyhedra.

It was mentioned above that the conic problem (3) can be considered as a special case of SIP problems. Therefore, to study the optimality in this problem, one can use the optimality conditions known from the theory of SIP. Notice that almost all known optimality conditions for SIP problems require the fulfillment of some additional conditions. As a rule, these conditions are either of the Slater type (see [7, 26, 28, 29] and the references therein) or include some weaker assumptions (see, e.g. [15, 16, 19]).

It worth to be mentioned that there is a small number of publications in the literature, where optimality conditions for set-semidefinite problems are formulated. These optimality conditions were also obtained under assumptions that in the case of problem (3), are equivalent to the Slater condition.

In [12], for a set-semidefinite problem in Banach and finite dimensional spaces, optimality conditions in a nondegenerate form were proved under a so-called Kurcyusz-Robinson-Zowe (KRZ) regularity condition (see (16) from [12]). For the problem in the form (3), this condition takes the form

$$
\Xi+\operatorname{cone}\left(\mathcal{C}^{K}-\{\mathcal{A}(\bar{x})\}\right)=\mathcal{S}(p),
$$

where $\bar{x} \in X, \Xi:=\left\{Z \in \mathcal{S}(p): Z=\sum_{k=1}^{n} A_{k} h_{k}, \forall h_{k} \in \mathbb{R}, k=1, \ldots, n\right\}$, and coneD stays for the conic hull of a set $\mathcal{D}$.

Let us prove that, for an affine matrix function $\mathcal{A}(x)$, condition (15) implies the Slater condition.

Suppose that condition (15) fulfills and let $\bar{S} \in \mathcal{S}(p)$ be such that $t^{\top} \bar{S} t<0$ for all $t \in \mathbb{R}^{p}, t \neq 0$. It follows from (15) that there exist $h=\left(h_{k} \in \mathbb{R}, k=1, \ldots, n\right)^{\top}, \alpha_{i} \geq 0, D_{i} \in \mathcal{C}^{K}, i \in \mathcal{I}$, such that

$$
\sum_{k=1}^{n} A_{k} h_{k}+\sum_{i \in \mathcal{I}} \alpha_{i}\left(D_{i}-\mathcal{A}(\bar{x})\right)=\bar{S} \Leftrightarrow \sum_{k=1}^{n} A_{k} h_{k}+(\tilde{D}-\alpha \mathcal{A}(\bar{x}))=\bar{S}
$$

with $\tilde{D}=\sum_{i \in \mathcal{I}} \alpha_{i} D_{i} \in \mathcal{C}^{K}, \alpha=\sum_{i \in \mathcal{I}} \alpha_{i} \geq 0$.

Suppose that $\alpha>0$. Then $\alpha \mathcal{A}(\bar{x}-h / \alpha)=\tilde{D}-\bar{S}$ and $\alpha t^{\top} \mathcal{A}(\bar{x}-h / \alpha) t=t^{\top} \tilde{D} t-t^{\top} \bar{S} t>0 \quad \forall t \in K, t \neq 0$. It follows from these relations that, for problem (3), the Slater condition holds true with $(\bar{x}-h / \alpha) \in X$.

Now suppose that $\alpha=0$ in (16). Then $\sum_{k=1}^{n} A_{k} h_{k}=\bar{S}$ and $\mathcal{A}(\bar{x}-h)=\mathcal{A}(\bar{x})-\bar{S}$. Hence

$$
t^{\top} \mathcal{A}(\bar{x}-h) t=t^{\top} \mathcal{A}(\bar{x}) t-t^{\top} \bar{S} t>0 \quad \forall t \in K, t \neq 0 .
$$

This implies that for problem (3), the Slater condition holds true with $(\bar{x}-h) \in X$.

The main contribution of this paper consists of new optimality criteria formulated in Theorems 1 and 2 . These criteria are CQ-free since they do not need any additional assumptions, whether it is the KRZ condition, or the Slater condition or any other CQ. This means that the scope of these optimality conditions is much wider than of other known conditions. This result was possible due to the application of the approach based on the concept of immobility. In section 6 , we will show how the obtained results can be used to produce strong dual formulations for problem (3) with a linear cost function.

\section{The properties of problem (8)}

To prove Theorem 1 we need some auxiliary results which will be considered in this section.

\subsection{A parametric representation of the cone $K$.}

First, consider the cone introduced in the previous section and used to identify the index sets of the equivalent problems (3) and (8):

$$
K=\left\{t \in \mathbb{R}^{p}: \mathbf{A} t=0, \mathbf{B} t \geq 0\right\} .
$$


Consider a subspace of $\mathbb{R}^{p}$ in the form

$$
H:=\left\{t \in \mathbb{R}^{p}: \mathbf{A} t=0, \mathbf{B} t=0\right\}
$$

and denote by $b_{s} \in \mathbb{R}^{p}, s \in S,|S| \leq p$, the vectors of an orthogonal basis of this subspace.

Consider the set

$$
H_{*}:=\left\{t \in \mathbb{R}^{p}: \mathbf{A} t=0, \mathbf{B} t \geq 0, t^{\top} b_{s}=0, s \in S\right\} .
$$

By construction, $H_{*}$ is a polyhedral convex cone. Let us show that $H_{*}$ is a pointed cone, i.e.

$$
t \neq 0, t \in H_{*} \Rightarrow-t \notin H_{*} .
$$

In fact, suppose that, on the contrary, there exists $\bar{t} \neq 0$ such that $\bar{t} \in H_{*}$ and $-\bar{t} \in H_{*}$. Then $\mathbf{A} \bar{t}=0, \mathbf{B} \bar{t}=0$, and, consequently, $\bar{t} \in H$. Thus we can conclude that $\bar{t}=\sum_{s \in S} \beta_{s} b_{s}$ for some scalars $\beta_{s}, s \in S$. As $\bar{t} \in H_{*}$, the following equalities should be satisfied:

$$
0=\bar{t}^{\top} b_{i}=\sum_{s \in S} \beta_{s} b_{s}^{\top} b_{i}=\beta_{i}, i \in S
$$

These equalities contradict the assumption that $0 \neq \bar{t}=\sum_{s \in S} \beta_{s} b_{s}$. The obtained contradiction proves that the convex polyhedral cone $H_{*}$ is pointed.

Denote by $a_{s}, s \in S_{*},\left|S_{*}\right|<\infty$, the extreme directions in the cone $H_{*}$. Recall that by the definition, the extreme directions in a polyhedral cone are its faces of dimension one.

The following lemma formulates the known result about representation of polyhedral cones.

Lemma 2

The cone $K$ defined in (17), can be written as follows:

$$
K=\left\{t \in \mathbb{R}^{p}: t=\sum_{s \in S} \beta_{s} b_{s}+\sum_{s \in S_{*}} \alpha_{s} a_{s}, \alpha_{s} \geq 0, s \in S_{*}\right\} .
$$

Note that the formula above is well consistent with the well-known fact that any nonempty convex set $C$ admits representation in the form $C=\operatorname{lin} C+\left(C \cap(\operatorname{lin} C)^{\perp}\right)$, where $\operatorname{lin} C$ is the largest subspace contained in the recession cone of $C$, the so-called lineality space of $C$ (see e.g. [25], p.65). Notice that for the polyhedral cone $K$ in the form (17), it holds $\operatorname{lin} K=H$ and $K \cap(\operatorname{lin} K)^{\perp}=H_{*}$, where $H$ and $H_{*}$ are defined in (18),(19).

\subsection{The properties of the set of immobile indices of problem (8)}

Here, as above, we consider a general case when the set of immobile indices of problem (8) may be nonempty.

\section{Proposition 1}

Suppose that for problem (8) it is satisfied: $X \neq \emptyset$ and $T_{i m} \neq \emptyset$. Then for any $x \in X$ and any $t \in T_{i m} \subset K$, the following relations hold true:

$$
a_{s}^{\top} \mathcal{A}(x) t \geq 0, s \in S_{*} ; b_{s}^{\top} \mathcal{A}(x) t=0, s \in S,
$$

where $\left\{b_{s} \in \mathbb{R}^{p}, s \in S,|S| \leq p\right\}$ is the orthogonal basis of the subspace $H$ defined in (18) and $\left\{a_{s}, s \in S_{*}\right.$, $\left.\left|S_{*}\right|<\infty\right\}$ is the set of extreme directions in the pointed cone $H_{*}$ defined in (19).

Proof. Suppose that, on the contrary, there exist $\bar{x} \in X$ and $\bar{t} \in T_{i m}$, such that one of two situations occurs:

$$
\text { 1. } \exists s_{0} \in S_{*} \text { such that } a_{s_{0}}^{\top} \mathcal{A}(\bar{x}) \bar{t}<0 \text { or } \quad \text { 2. } \exists s_{0} \in S \text { such that } b_{s_{0}}^{\top} \mathcal{A}(\bar{x}) \bar{t} \neq 0 \text {. }
$$

Let us assume, first, that situation 1 has happened. By construction, the vector $a_{s_{0}}$ is a recession direction for $\bar{t}$ in the cone $K: t(\theta):=\bar{t}+\theta a_{s_{0}} \in K \quad \forall \theta \geq 0$. 
Let us compute $(t(\theta))^{\top} \mathcal{A}(\bar{x}) t(\theta)=2 \theta a_{s_{0}}^{\top} \mathcal{A}(\bar{x}) \bar{t}+\theta^{2} a_{s_{0}}^{\top} \mathcal{A}(\bar{x}) a_{s_{0}}$. Taking into account that $a_{s_{0}}^{\top} \mathcal{A}(\bar{x}) \bar{t}<0$, one can conclude from the latter equality that there exists $\bar{\theta}>0$ such that $(t(\bar{\theta}))^{\top} \mathcal{A}(\bar{x}) t(\bar{\theta})<0$. The latter inequality implies the inequality

$$
\widetilde{t}^{\top} \mathcal{A}(\bar{x}) \widetilde{t}<0, \text { where } \tilde{t}:=t(\bar{\theta}) /\|t(\bar{\theta})\|_{\infty} \in T,
$$

that contradicts the condition $\bar{x} \in X$.

Now suppose that the situation 2 has occurred. Set $\gamma:=b_{s_{0}}^{\top} \mathcal{A}(\bar{x}) \bar{t}$. By construction, the direction $-\gamma b_{s_{0}}$ is feasible for $\bar{t}$ in $K: t(\theta):=\bar{t}-\theta \gamma b_{s_{0}} \in K \quad \forall \theta \geq 0$. By simple computations, we get

$$
(t(\theta))^{\top} \mathcal{A}(\bar{x}) t(\theta)=-2 \theta \gamma b_{s_{0}}^{\top} \mathcal{A}(\bar{x}) \bar{t}+\theta^{2} \gamma^{2} b_{s_{0}}^{\top} \mathcal{A}(\bar{x}) b_{s_{0}}=-2 \theta \gamma^{2}+\theta^{2} \gamma^{2} b_{s_{0}}^{\top} \mathcal{A}(\bar{x}) b_{s_{0}},
$$

and taking into account the inequality $\gamma \neq 0$, one can conclude that there exists $\bar{\theta}>0$ such that $t^{\top}(\bar{\theta}) \mathcal{A}(\bar{x}) t(\bar{\theta})<0$. But this inequality implies relations (21) and hence contradicts the condition $\bar{x} \in X$. The proposition is proved.

\section{Lemma 3}

Given convex SIP problem (8), the immobile index set $T_{i m}$ is either empty or can be represented as a union of a finite number of convex closed bounded polyhedra.

Proof. If $T_{i m}=\emptyset$, the lemma is evident.

Suppose that $T_{i m} \neq \emptyset$. Consider any $t \in T_{i m} \subset K$. It follows from Lemma 2 that $t$ admits the representation

$$
t=\sum_{s \in S} \beta_{s} b_{s}+\sum_{s \in S_{*}} \alpha_{s} a_{s}, \alpha_{s} \geq 0, s \in S_{*} .
$$

For $t \in T_{i m}$, denote

$$
S_{*}^{+}(t):=\left\{s \in S_{*}: \alpha_{s}>0\right\}
$$

Set

$$
\mathcal{Z}:=\left\{Z \subset S_{*}: \exists t \in T_{i m}, Z=S_{*}^{+}(t)\right\} .
$$

From the definition of the set $\mathcal{Z}$, it follows that it is finite: $|\mathcal{Z}| \leq 2^{\left|S_{*}\right|}$, where $2^{\left|S_{*}\right|}<\infty$ is the number of all subsets of the finite set $S_{*}$.

For any $Z \in \mathcal{Z}$, consider the corresponding set

$$
\widetilde{T}(Z):=\operatorname{cl}\left\{t \in T_{i m}: S_{*}^{+}(t)=Z\right\},
$$

where $\operatorname{cl}(\mathcal{D})$ stays for the closure of a set $\mathcal{D} \subset \mathbb{R}^{p}$.

It is evident that

$$
T_{i m}=\bigcup_{Z \in \mathcal{Z}} \widetilde{T}(Z)
$$

By construction, for any $\bar{t} \in \widetilde{T}(Z)$, we have $\bar{t} \in T_{i m}, S_{*}^{+}(\bar{t}) \subset Z$, and

$$
\bar{t}=\lim _{\omega \rightarrow \infty} t(\omega), \quad \text { where } t(\omega) \in T_{i m}, \quad S_{*}^{+}(t(\omega))=Z .
$$

Let us show that for any $\bar{t} \in \widetilde{T}(Z)$, we have

$$
a_{s}^{\top} \mathcal{A}(x) \bar{t}=0 \forall s \in Z \quad \forall x \in X .
$$

Suppose the contrary. Then for some $\bar{t} \in \widetilde{T}(Z)$, taking into account Proposition 1, there exist $s_{0} \in Z$ and $\bar{x} \in X$, such that $a_{s_{0}}^{\top} \mathcal{A}(\bar{x}) \bar{t}>0$. From the latter inequality and relations (23), we conclude that there exists $t(\omega) \in T_{\text {im }}$ such that $S_{*}^{+}(t(\omega))=Z$ and

$$
a_{s_{0}}^{\top} \mathcal{A}(\bar{x}) t(\omega)>0 .
$$

Since $S_{*}^{+}(t(\omega))=Z$ and $s_{0} \in Z$, the direction $-a_{s_{0}}$ is feasible for $t(\omega)$ in $K$, i.e. there exists $\theta_{0}>0$ such that

$$
t(\theta):=t(\omega)-\theta a_{s_{0}} \in K \quad \forall \theta \in\left[0, \theta_{0}\right] .
$$




\section{Compute}

$$
(t(\theta))^{\top} \mathcal{A}(\bar{x}) t(\theta)=-2 \theta a_{s_{0}}^{\top} \mathcal{A}(\bar{x}) t(\omega)+\theta^{2} a_{s_{0}}^{\top} \mathcal{A}(\bar{x}) a_{s_{0}} .
$$

From the latter equality and inequality (25), it follows that there exists $0<\bar{\theta} \leq \theta_{0}$ such that $(t(\theta))^{\top} \mathcal{A}(\bar{x}) t(\bar{\theta})<0$. But this inequality implies relations (21) that contradict the assumption $\bar{x} \in X$. Hence equalities (24) are proved.

Now let us show that

$$
\widehat{t}^{\top} \mathcal{A}(x) \bar{t}=0 \quad \forall \widehat{t} \in \widetilde{T}(Z), \quad \forall \bar{t} \in \widetilde{T}(Z), \quad \forall x \in X .
$$

In fact, any vector $\widehat{t} \in \widetilde{T}(Z)$ can be written as follows:

$$
\widehat{t}=\sum_{s \in S} \beta_{s} b_{s}+\sum_{s \in Z} \alpha_{s} a_{s}
$$

Taking into account this representation, equalities (24) and relations (20), we obtain

$$
\widehat{t}^{\top} \mathcal{A}(x) \bar{t}=\left(\sum_{s \in S} \beta_{s} b_{s}+\sum_{s \in Z} \alpha_{s} a_{s}\right)^{\top} \mathcal{A}(x) \bar{t}=0 \quad \forall x \in X .
$$

Consequently, we have proved equalities (26).

Given $Z \in \mathcal{Z}$, let $0<r=\operatorname{dim} \operatorname{span}(\widetilde{T}(Z)) \leq p$, being here $\operatorname{span}(\mathcal{D})$ the linear subspace generated by $\mathcal{D} \subset \mathbb{R}^{p}$. Let $\left\{t^{1}, \ldots, t^{r}\right\} \subset \widetilde{T}(Z)$ be a basis of $\operatorname{span}(\widetilde{T}(Z))$. Denote

$$
\widehat{T}(Z):=\left\{t \in \mathbb{R}^{p}: t=\sum_{i \in I} t^{i} \gamma_{i}, \mathbf{B} t \geq 0,\|t\|_{\infty}=1\right\}, \text { where } I:=\{1, \ldots, r\} .
$$

It is easy to see that the set $\widehat{T}(Z)$ is a union of a finite number of convex bounded polyhedra.

Suppose that $t \in \widetilde{T}(Z)$. Hence, by construction, $B t \geq 0,\|t\|_{\infty}=1$ and $t$ admits a representation $t=\sum_{i \in I} t^{i} \gamma_{i}$. Hence $t \in \widehat{T}(Z)$ and $\widetilde{T}(Z) \subset \widehat{T}(Z)$. Consequently

$$
\bigcup_{Z \in \mathcal{Z}} \widetilde{T}(Z)=T_{i m} \subset \bigcup_{Z \in \mathcal{Z}} \widehat{T}(Z)
$$

Now consider any $t \in \widehat{T}(Z)$. By construction, we have $\mathbf{A} t=0, \mathbf{B} t \geq 0,\|t\|_{\infty}=1$, and, consequently, $t \in T$. Since $t^{i} \in \widetilde{T}(Z), i \in I$, it follows from (26) that

$$
\left(t^{i}\right)^{\top} \mathcal{A}(x) t^{j}=0, i \in I, j \in I, \forall x \in X .
$$

Taking into account these equalities, for any $x \in X$, we get

$$
t^{\top} \mathcal{A}(x) t=\sum_{i \in I} \sum_{j \in I} \gamma_{i} \gamma_{j}\left(t^{i}\right)^{\top} \mathcal{A}(x) t^{j}=0 .
$$

This implies that $t \in T_{i m}$ and hence $\widehat{T}(Z) \subset T_{i m}$. Consequently, $\bigcup_{Z \in \mathcal{Z}} \widehat{T}(Z) \subset T_{i m}$. This inclusion together with (27) imply

$$
\bigcup_{Z \in \mathcal{Z}} \widehat{T}(Z)=T_{i m}
$$

Taking into account that $|\mathcal{Z}|<\infty$ and the fact that each set $\widehat{T}(Z)$ is a union of a finite number of convex bounded polyhedra, we conclude from the latter equality that the statement of the lemma is proved.

Consider the set $\left\{t^{*}(j), j \in J\right\}$ of vertices of the polyhedron conv $T_{i m}$. Recall that in the case $T_{i m}=\emptyset$, we have conv $T_{i m}=\emptyset$ and $J=\emptyset$. 
Suppose that $T_{i m} \neq \emptyset$. Since $t^{*}(j) \in T_{i m}$ for any $j \in J$, it follows from Proposition 1 that the following relations hold true:

$$
a_{s}^{\top} \mathcal{A}(x) t^{*}(j) \geq 0, s \in S_{*} ; b_{s}^{\top} \mathcal{A}(x) t^{*}(j)=0, s \in S, j \in J, \forall x \in X .
$$

Denote

$$
\mathcal{X}:=\left\{x \in \mathbb{R}^{n}: a_{s}^{\top} \mathcal{A}(x) t^{*}(j) \geq 0, s \in S_{*} ; b_{s}^{\top} \mathcal{A}(x) t^{*}(j)=0, s \in S, j \in J\right\} .
$$

Evidently, $X \subset \mathcal{X}$.

\section{Proposition 2}

Consider the SIP problem (8) with the set of immobile indices $T_{i m}$. Let the set $\mathcal{X}$ be defined in (29). Then the following relations hold true:

$$
t^{\top} \mathcal{A}(x) t \geq 0 \quad \forall t \in \operatorname{conv} T_{i m}, \quad \forall x \in \mathcal{X} .
$$

Proof. Let $t \in \operatorname{conv} T_{i m}$. Then $t=\sum_{j \in J} \gamma_{j} t^{*}(j), \sum_{j \in J} \gamma_{j}=1, \gamma_{j} \geq 0, j \in J$. Hence for any $x$, it holds

$$
t^{\top} \mathcal{A}(x) t=\left(\sum_{j \in J} \gamma_{j} t^{*}(j)\right)^{\top} \mathcal{A}(x)\left(\sum_{j \in J} \gamma_{j} t^{*}(j)\right)=\sum_{i \in J} \sum_{j \in J} \gamma_{i} \gamma_{j}\left(t^{*}(i)\right)^{\top} \mathcal{A}(x) t^{*}(j) .
$$

Let us show that for any $i \in J, j \in J$,

$$
\left(t^{*}(i)\right)^{\top} \mathcal{A}(x) t^{*}(j) \geq 0, \quad \forall x \in \mathcal{X}
$$

In fact, since the vector $t^{*}(i)$ belongs to $K$, it admits a representation

$$
t^{*}(i)=\sum_{s \in S} \beta_{s i} b_{s}+\sum_{s \in S_{*}} \alpha_{s i} a_{s}, \quad \text { where } \alpha_{s i} \geq 0, s \in S_{*} .
$$

Hence, taking into account (29), we get

$$
\left(t^{*}(i)\right)^{\top} \mathcal{A}(x) t^{*}(j)=\left(\sum_{s \in S} \beta_{s i} b_{s}+\sum_{s \in S_{*}} \alpha_{s i} a_{s}\right)^{\top} \mathcal{A}(x) t^{*}(j)=\sum_{s \in S_{*}} \alpha_{s i} a_{s}^{\top} \mathcal{A}(x) t^{*}(j) \geq 0, \quad \forall x \in \mathcal{X} .
$$

Inequalities (30) follow from (31) and (32). The proposition is proved.

\subsection{The properties of the feasible set of problem (8)}

In this section, we will obtain some properties of the feasible set of problem (8). These properties are closely connected with the properties of the elements of the convex hull of the set of normalized immobile indices in this problem.

Denote

$$
\begin{gathered}
T(\varepsilon):=\left\{t \in T, \rho\left(t, \operatorname{conv} T_{i m}\right) \geq \varepsilon\right\}, \widehat{T(\varepsilon)}:=\left\{t \in T, \rho\left(t, \operatorname{conv} T_{i m}\right) \leq \varepsilon\right\}, \\
X(\varepsilon):=\left\{x \in \mathcal{X}: t^{\top} \mathcal{A}(x) t \geq 0 \forall t \in T(\varepsilon)\right\},
\end{gathered}
$$

where $\varepsilon>0$, the set $\mathcal{X}$ is defined in (29), the distance between a vector $l$ and a set $B$ in $\mathbb{R}^{p}$ is defined as $\rho(l, B)=\min _{\tau \in B}\|l-\tau\|$, and $\|a\|$ stays for a norm of vector $a \in \mathbb{R}^{p}$.

Lemma 4

Consider the SIP problem (8) with the set of immobile indices $T_{i m}$ and the feasible set $X$. There exists $\varepsilon_{0}>0$ such that $X\left(\varepsilon_{0}\right)=X$, where the set $X(\varepsilon)$ is defined in (34). 
Proof. It is evident that $X \subset X(\varepsilon)$ for all $\varepsilon>0$.

Let us show that there exists $\varepsilon_{0}>0$ such that $X\left(\varepsilon_{0}\right) \subset X$. Suppose the contrary. Then for all $\varepsilon>0$ there exists $x(\varepsilon) \in X(\varepsilon)$ such that

$$
(t(\varepsilon))^{\top} \mathcal{A}(x(\varepsilon)) t(\varepsilon)<0,
$$

where $t(\varepsilon) \in \arg \left\{\min _{t} t^{\top} \mathcal{A}(x(\varepsilon)) t\right.$, s.t. $\left.t \in T\right\}$.

It is easy to prove that $t(\varepsilon)$ is also an optimal solution of the problem

$$
\text { minimize } t^{\top} \mathcal{A}(x(\varepsilon)) t \text {, subject to } t \in \widetilde{T},
$$

where $\widetilde{T}:=\left\{t \in K:\|t\|_{\infty} \leq 1\right\}$. Notice that $T \subset \widetilde{T}$, and the set $\widetilde{T}$ is convex. By construction (see Proposition 2 and definition (34)), it holds

$$
t^{\top} \mathcal{A}(x(\varepsilon)) t \geq 0, \forall t \in T(\varepsilon) \cup \operatorname{conv} T_{i m} .
$$

Hence we can conclude that $t(\varepsilon) \in \widehat{T(\varepsilon)} \backslash \operatorname{conv} T_{i m}$ and there exists a limiting point $t^{*}$ of the sequence $t(\varepsilon), \varepsilon \rightarrow 0$ such that $t^{*} \in \operatorname{conv} T_{i m} \subset T$. To simplify notation without loss of generality suppose that $t^{*}=\lim _{\varepsilon \rightarrow 0} t(\varepsilon)$.

Set

$$
P^{ \pm}(\varepsilon):=\left\{k \in\{1, \ldots, p\}: t_{k}(\varepsilon)= \pm 1\right\}, N^{0}(\varepsilon):=\left\{k \in\left\{1, \ldots, m_{*}\right\}: B_{k}^{\top} t(\varepsilon)=0\right\},
$$

where $t_{k}(\varepsilon)$ denotes the $k$-th component of the vector $t(\varepsilon)$ and $B_{k}^{\top}$ stays for the $k$-th raw of the matrix $\mathbf{B} \in \mathbb{R}^{m_{*} \times p}$.

Since the sets $\{1, \ldots, p\}$ and $\left\{1, \ldots, m_{*}\right\}$ are finite, there exists a sequence $\varepsilon_{k}>0, k=1,2, \ldots$ and sets $P^{ \pm}, N^{0}$ such that $\lim _{k \rightarrow \infty} \varepsilon_{k}=0, P^{ \pm}\left(\varepsilon_{k}\right)=P^{ \pm}$, and $N^{0}\left(\varepsilon_{k}\right)=N^{0}$ for sufficiently large $k>0$. To simplify the designation, without loss of generality, we can consider that

$$
P^{ \pm}(\varepsilon)=P^{ \pm} \text {and } N^{0}(\varepsilon)=N^{0} \text { for sufficiently small } \varepsilon>0 .
$$

Given a sufficiently small $\varepsilon>0$, consider vector $l(\varepsilon):=t(\varepsilon)-t^{*}$. It is easy to see that due to the convexity of the set $\widetilde{T}$, we have $t^{*}+\lambda\left(t(\varepsilon)-t^{*}\right)=t^{*}+\lambda l(\varepsilon) \in \widetilde{T}$ for all $\lambda \in[0,1]$. Hence $l(\varepsilon)$ is a feasible direction for $t^{*}$ in $\widetilde{T}$.

Since by construction, $\mathbf{A} l(\varepsilon)=0$, then, according to definition, the direction $l(\varepsilon)$ will be feasible for $t(\varepsilon)$ in the set $\widetilde{T}$ if there exists $\theta_{0}>0$ such that $-1 \leq t_{k}(\varepsilon)+\theta l_{k}(\varepsilon) \leq 1, k=1, \ldots, p$, and $B(t(\varepsilon)+\theta l(\varepsilon)) \geq 0$, for all $\theta \in\left[0, \theta_{0}\right]$. Hence the direction $l(\varepsilon)$ is feasible for $t(\varepsilon)$ in the set $\widetilde{T}$ if

$$
l_{k}(\varepsilon) \leq 0 \text { for } k \in P^{+} ; \quad l_{k}(\varepsilon) \geq 0 \text { for } k \in P^{-} ; \quad B_{k}^{\top} l(\varepsilon) \geq 0 \text { for } k \in N^{0} .
$$

The relations above are fulfilled since it follows from (37) that $l_{k}(\varepsilon):=t_{k}(\varepsilon)-t_{k}^{*}=0, k \in P^{+} \cup P^{-}$and $B_{k}^{\top} l(\varepsilon)=B_{k}^{\top}\left(t_{k}(\varepsilon)-t_{k}^{*}\right)=0$ for $k \in N^{0}$. Consequently, the direction $l(\varepsilon)$ is feasible for both vectors, $t^{*}$ and $t(\varepsilon)$, in the set $\widetilde{T}$ and, hence,

$$
\bar{t}(\sigma):=t^{*}+\sigma l(\varepsilon)=t^{*}+\sigma\left(t(\varepsilon)-t^{*}\right) \in \widetilde{T}, \quad \forall \sigma \in\left[0, \sigma_{0}\right], \text { where } \sigma_{0}>1 .
$$

For a fixed $\varepsilon>0$ that satisfies the assumptions above, define the function

$$
\begin{gathered}
w(\sigma):=(\bar{t}(\sigma))^{\top} \mathcal{A}(x(\varepsilon)) \bar{t}(\sigma)=\left(t^{*}\right)^{\top} \mathcal{A}(x(\varepsilon)) t^{*}+2 \sigma(l(\varepsilon))^{\top} \mathcal{A}(x(\varepsilon)) t^{*}+\sigma^{2}(l(\varepsilon))^{\top} \mathcal{A}(x(\varepsilon)) l(\varepsilon) \\
=a \sigma^{2}+2 b \sigma+c, \quad \sigma \in\left[0, \sigma_{0}\right],
\end{gathered}
$$

where $c:=\left(t^{*}\right)^{\top} \mathcal{A}(x(\varepsilon)) t^{*}, b:=(l(\varepsilon))^{\top} \mathcal{A}(x(\varepsilon)) t^{*}, a:=(l(\varepsilon))^{\top} \mathcal{A}(x(\varepsilon)) l(\varepsilon)$.

By construction, for $\sigma^{*}=1$ we have $\bar{t}\left(\sigma^{*}\right)=t(\varepsilon)$. Then $w\left(\sigma^{*}\right)=(t(\varepsilon))^{\top} \mathcal{A}(x(\varepsilon)) t(\varepsilon)$, and $w\left(\sigma^{*}\right)$ is the optimal value of the cost function of the problem (36). Hence

$$
w\left(\sigma^{*}\right)=\min _{\sigma \in\left[0, \sigma_{0}\right]} w(\sigma)=\min _{\sigma \in\left[0, \sigma_{0}\right]}\left(a \sigma^{2}+2 b \sigma+c\right) .
$$


As $\sigma^{*} \in\left(0, \sigma_{0}\right)$, one can conclude from (38) that $2 a \sigma^{*}+2 b=2 a+2 b=0$. Then $-a=b$ and we get the following equivalent equalities:

$$
\begin{aligned}
-(l(\varepsilon))^{\top} \mathcal{A}(x(\varepsilon)) t^{*} & =(l(\varepsilon))^{\top} \mathcal{A}(x(\varepsilon)) l(\varepsilon) \Longleftrightarrow \\
(t(\varepsilon))^{\top} \mathcal{A}(x(\varepsilon)) t^{*} & =(t(\varepsilon))^{\top} \mathcal{A}(x(\varepsilon)) t(\varepsilon) .
\end{aligned}
$$

By construction, $t^{*} \in \operatorname{conv} T_{i m}$. Therefore $t^{*}=\sum_{j \in J} \gamma_{j} t^{*}(j), \quad \sum_{j \in J} \gamma_{j}=1, \gamma_{j} \geq 0, j \in J$.

Since $t(\varepsilon) \in \widetilde{T} \subset K$, then $t(\varepsilon)=\sum_{s \in S} \beta_{s} b_{s}+\sum_{s \in S_{*}} \alpha_{s} a_{s}$, where $\alpha_{s} \geq 0, s \in S_{*}$. Consequently, taking into account the inclusion $x(\varepsilon) \in X(\varepsilon) \subset \mathcal{X}$, where $\mathcal{X}$ is defined in (29), we have

$$
\begin{aligned}
(t(\varepsilon))^{\top} \mathcal{A}(x(\varepsilon)) t^{*} & =\left(\sum_{s \in S} \beta_{s} b_{s}+\sum_{s \in S_{*}} \alpha_{s} a_{s}\right)^{\top} \mathcal{A}(x(\varepsilon)) \sum_{j \in J} \gamma_{j} t^{*}(j) \\
& =\sum_{s \in S_{*}} \sum_{j \in J} \alpha_{s} \gamma_{j} a_{s}^{\top} \mathcal{A}(x(\varepsilon)) t^{*}(j) \geq 0 .
\end{aligned}
$$

The latter inequality and inequality (35) contradict the equality (39). The lemma is proved.

The next lemma can be proved using the same reasoning scheme as in the proof of Lemma 3 from [20].

\section{Lemma 5}

Given SIP problem (8) with the set of immobile indices $T_{i m}$, for any $\varepsilon>0$ there exists a vector $x(\varepsilon) \in \mathcal{X}$ such that

$$
t^{\top} \mathcal{A}(x(\varepsilon)) t>0 \quad \forall t \in T(\varepsilon),
$$

where the sets $\mathcal{X}$ and $T(\varepsilon)$ are defined in (29), (33).

\section{The proof of Theorem 1}

We will prove here Theorem 1 for problem (8). Taking into account that problems (3) and (8) are equivalent, the theorem will be valid also for the $K$-semidefinite problem (3).

Necessity. Let $x^{0} \in X$ be an optimal solution of the problem. Choose $\varepsilon_{0}>0$ as in Lemma 4 and consider a perturbed SIP problem

$$
\text { minimize } c(x) \text {, subject to } x \in X\left(\varepsilon_{0}\right),
$$

where the set $X(\varepsilon)$ is defined in (34). This problem can be rewritten as follows:

$$
\begin{array}{ll} 
& \operatorname{minimize} c(x), \\
\text { subject to } & x \in \mathbb{R}^{n}, t^{\top} \mathcal{A}(x) t \geq 0 \quad \forall t \in T\left(\varepsilon_{0}\right), \\
& a_{s}^{\top} \mathcal{A}(x) t^{*}(j) \geq 0, s \in S_{*} ; b_{s}^{\top} \mathcal{A}(x) t^{*}(j)=0, s \in S, j \in J .
\end{array}
$$

According to Lemma 4, the set of feasible solutions of problem (41) coincides with the set of feasible solutions of the original problem (8). Hence vector $x^{0}$ is an optimal solution of the perturbed problem (41) as well.

Problem (41) has the following important properties:

- the index set $T\left(\varepsilon_{0}\right)$ is compact,

- the constraints satisfy the Slater type condition (see Lemma 5).

Hence, applying the classic optimality conditions (see, for example, Theorem 5.107 in [7]) for the optimal solution $x^{0}$ of problem (41), we can state that there exist numbers and vectors

$$
y(i)>0, \quad t(i) \in T\left(\varepsilon_{0}\right), i \in I,|I| \leq n ; \quad \alpha_{s j} \geq 0, s \in S_{*}, \beta_{s j} \in \mathbb{R}, s \in S, j \in J,
$$


such that the following relations hold:

$$
\begin{gathered}
-\frac{\partial c\left(x^{0}\right)}{\partial x_{k}}+\sum_{i \in I} y(i)(t(i))^{\top} A_{k} t(i)+\sum_{j \in J}\left(\sum_{s \in S_{*}} \alpha_{s j} a_{s}+\sum_{s \in S} \beta_{s j} b_{s}\right)^{\top} A_{k} t^{*}(j)=0, k=1, \ldots, n \\
(t(i))^{\top} \mathcal{A}\left(x^{0}\right) t(i)=0, i \in I ; \quad \alpha_{s j} a_{s}^{\top} \mathcal{A}\left(x^{0}\right) t^{*}(j)=0, s \in S_{*}, j \in J .
\end{gathered}
$$

Denote $\tau(i):=\sqrt{y(i)} t(i), i \in I, \lambda(j):=\frac{1}{2}\left(\sum_{s \in S_{*}} \alpha_{s j} a_{s}+\sum_{s \in S} \beta_{s j} b_{s}\right) \in K, j \in J$. It is easy to see that relations (43), (44) can be written in the form (11) with matrices $U$ and $V$ defined in (12). The necessity is proved.

Sufficiency. Suppose that for $x^{0} \in X$, there exist vectors (10) such that for matrices (12) the relations (11) hold true. Then it is easy to show that the vector $x^{0}$ is an optimal solution in the following convex problem with linear constraints:

$$
\begin{aligned}
& \operatorname{minimize} c(x) \\
& (\mathrm{CP}): \quad \text { subject to } x \in \mathbb{R}^{n},(\tau(i))^{\top} \mathcal{A}(x) \tau(i) \geq 0, i \in I, \\
& \\
& a_{s}^{\top} \mathcal{A}(x) t^{*}(j) \geq 0, s \in S_{*} ; b_{s}^{\top} \mathcal{A}(x) t^{*}(j)=0, s \in S, j \in J .
\end{aligned}
$$

It is evident that the feasible set $X$ of problem (8) belongs to the set of feasible solutions of the problem (CP). Hence the optimality of $x^{0} \in X$ in (CP) implies the optimality of $x^{0}$ in the original problem (8). The theorem is proved.

\section{Dual formulations for the linear $K$-semidefinite problem (3): standard Lagrangian and regularized dual problems}

In this section, we will suppose that the cost function of the special conic problem (3) is linear and discuss some new dual formulations for this problem.

In our papers [17] and [20], we derived regularized primal and dual formulations for linear copositive problems. These formulations are explicit and guarantee the strong duality. In this section, in a similar way, we will derive new strong dual formulations for the $K$-semidefinite problem (3). To obtain these results, we will use Theorem 2.

Consider a $K$-semidefinite problem with linear cost function in the form

$$
\text { minimize } c^{\top} x \text { subject to } \mathcal{A}(x) \in \mathcal{C}^{K}, x \in \mathbb{R}^{n},
$$

where $c=\left(c_{1}, c_{2}, \ldots, c_{n}\right)$, function $\mathcal{A}(x)$ and the polyhedral set $K$ are defined as in (3).

First of all, let us formulate for problem (45), the (standard) Lagrangian dual problem. According to [24], it has the form:

$$
\begin{gathered}
\operatorname{maximize}\left(-W \bullet A_{0}\right), \\
\text { subject to }-c_{k}+W \bullet A_{k}=0, k=1,2, \ldots, n ; W \in\left(\mathcal{C}^{K}\right)^{*},
\end{gathered}
$$

where the cone $\left(\mathcal{C}^{K}\right)^{*}=\operatorname{conv}\left\{l l^{\top}: l \in K\right\}$ is the dual cone to the cone $\mathcal{C}^{K}$.

It is a known fact (see e.g.[24]) that if the constraints of problem (45) satisfy the Slater condition, then there is no gap between the optimal values of this problem and its Lagrangian dual.

If the constraints of problem (45) do not satisfy the Slater condition, then the positive gap is possible even in the case when the primal problem has an optimal solution.

Consider the following cones:

$$
\mathcal{C}^{K}(j):=\left\{D \in \mathcal{S}(p): t^{\top} D t^{*}(j) \geq 0 \quad \forall t \in K\right\}, \quad j \in J .
$$


These cones are explicitly generated by the finite set $\left\{t^{*}(j), j \in J\right\}$ of vertices of the polyhedron conv $T_{i m}$, where $T_{i m}$ is the normalized immobile index set in problem (45).

One can show that for any $j \in J$, the cone $\mathcal{C}^{K}(j)$ is convex and closed and the corresponding dual cone has the form

$$
\left(\mathcal{C}^{K}(j)\right)^{*}=\left\{t\left(t^{*}(j)\right)^{\top}+t^{*}(j) t^{\top}: t \in K\right\} .
$$

Denote

$$
\mathcal{K}:=\left(\bigcap_{j \in J} \mathcal{C}^{K}(j)\right) \cap \mathcal{C}^{K}
$$

Evidently, $\mathcal{K} \subset \mathcal{C}^{K}$, where the cone $\mathcal{C}^{K}$ is defined in (1). It can be shown (see e.g. [4, 6]) that the dual cone to $\mathcal{K}$ has the form

$$
\mathcal{K}^{*}=\operatorname{cl}\left(\sum_{j \in J}\left(\mathcal{C}^{K}(j)\right)^{*}+\left(\mathcal{C}^{K}\right)^{*}\right)
$$

Hence

$$
\mathcal{K}^{*}=\operatorname{cl}\left\{W \in \mathcal{S}(p): W=U+V, U \in\left(\mathcal{C}^{K}\right)^{*}, V \in \mathcal{V}(p)\right\},
$$

where $\mathcal{V}(p)$ is defined in (13).

Taking into account Proposition 1, one can show that the $K$-semidefinite problem (45) can be rewritten in the conic form

$$
\text { minimize } c^{\top} x \text {, subject to } x \in \mathbb{R}^{n}, \mathcal{A}(x) \in \mathcal{K} \subset \mathcal{C}^{K} .
$$

Let us refer to problem (48) as a regularized primal (set-semidefinite) problem. The dual problem to (48) (the regularized dual problem) has the form

$$
\begin{gathered}
\operatorname{maximize}\left(-W \bullet A_{0}\right), \\
\text { subject to }-c_{k}+W \bullet A_{k}=0 \quad \forall k=1,2, \ldots, n, W \in \mathcal{K}^{*} .
\end{gathered}
$$

It is easy to show that, given any feasible solution $x$ of problem (48) and any feasible solution $W$ of the dual problem (49), the following inequality (weak duality) is satisfied:

$$
c^{\top} x \geq-W \cdot A_{0} .
$$

Moreover, taking into account Theorem 2 and representation (47), we can conclude that for any optimal solution $x^{0}$ of problem (48), there exists a feasible solution $W^{0}=U^{0}+V^{0}, U^{0} \in\left(\mathcal{C}^{K}\right)^{*}, V^{0} \in \mathcal{V}(p)$, of the dual problem (49) such that the strong duality property holds:

$$
c^{\top} x^{0}=-W^{0} \bullet A_{0} .
$$

This conclusion implies the following proposition.

\section{Proposition 3}

Suppose that the $K$-semidefinite problem (45) has an optimal solution. Then

- an optimal solution of the regularized dual problem (49) exists, and

- there is no gap between the optimal values of problem (45) (equivalently, the regularized primal $K$ semidefinite problem (48)), and its regularized dual problem (49).

From this proposition, it follows that the strong duality is guaranteed for the primal problem (45) and its regularized dual problem (49), while, as it was mentioned above, for the pair constituted by the primal problem (45) and its (standard) Lagrangian dual problem (46), the strong duality may fail.

The main contribution of this section is to obtain for the set-semidefinite problem (45) a regularized dual one (49) satisfying the strong duality conditions without CQs or any other assumptions. The dual problem (49) has an explicit form and is constructed using the vertices of the convex hull of the normalized immobile index set of the constraints in the primal problem. 
In $[24,30]$, a CQ-free duality theory for conic optimization was developed in terms of the so-called minimal cone. Being quite general, this theory has one disadvantage in terms of its application, namely, it is very abstract.

To the best of our knowledge, for problem (45), there are no other explicit CQ-free dual formulations satisfying strong duality relations.

We plan to dedicate a special paper to the detailed comparison of the new conic formulations obtained using the notion of the immobile indices with that from $[24,30]$.

\section{Conclusions}

The main contribution of the paper consists in the formulation of new CQ-free optimality conditions and strong duality results for set-semidefinite programming problems. To obtain these results, we used the approach, suggested in our previous papers for convex SIP, SDP, and copositive problems, and based on the concept of immobile indices.

The main conclusions we can draw from the results of the paper, are the following.

- The concept of immobile indices being applied to set-semidefinite optimization permits one to obtain new optimality conditions.

- The set of immobile indices has a special structure and its study is important. Notice that the optimality conditions formulated in Theorems 1 and 2 do not use the proper normalized immobile indices but are formulated with the help of a finite number of vertices of the convex hull of the normalized immobile index set. In the future, we plan to obtain CQ-free optimality conditions and strong duality results without explicit use of there vertices as well.

- The optimality conditions and strong duality relations obtained in the paper for the special set-semidefinite problems may be used to develop numerical methods for solving these problems.

\section{Acknowledgements}

This work was partially supported by state research program "Convergence"(Republic Belarus), Task 1.3.01, by Portuguese funds through CIDMA - Center for Research and Development in Mathematics and Applications, and FCT - Portuguese Foundation for Science and Technology, within the project UID/MAT/04106/2019.

The authors are grateful to the anonymous reviewers for careful reading of the paper and for their constructive comments and advices.

\section{References}

1. Ahmed F., Dür M., Still G. Copositive Programming via Semi-Infinite Optimization, J. Optim. Theory Appl., 159, pp. 322-340, 2013.

2. Anjos M.F., Lasserre J.B. Eds. Handbook of Semidefinite, Conic and Polynomial Optimization: Theory, Algorithms, Software and Applications, International Series in Operational Research and Management Science, 166 p., Springer,2012

3. Asprey S.P., Maccietto S. Eds. Dynamic model development: methods, theory and applications, In: Proceedings of the Workshop on the Life af a Process Model-From Conception to Action, Imperial Colledge, London, UK, October 25-26, 2000.

4. Berman A., Shaked-Monderer N. PCompletely Positive Matrices, World Scientific, 2003, 216 p.

5. Bomze I.M. Copositive optimization - Recent developments and applications, EJOR 216(3), pp. 509-520, 2012.

6. Bomze I.M., Dür M., de Klerk E., Roos C., Quist A.J., Terlaky T. On copositive programming and standard quadratic optimization problems, Journal Global Optim. 18, pp. 301-320, 2000.

7. Bonnans J.F., Shapiro A. Perturbation analysis of optimization problems, Springer-Verlag, New-York, 2000.

8. Boyd S. P., Vandenberghe L. Convex Optimization, Cambridge University Press, 2004.

9. de Klerk E., Pasechnik D.V. Approximation of the stability number of a graph via copositive programming, SIAM J. Optim., 12, pp. 875-892, 2002.

10. Dür M. Copositive Programming - a Survey, In: Diehl M, Glineur F, Jarlebring E, Michielis W., editors. Recent advances in Optimization and its applications in Engineering; Springer-Verlag Berlin Heidelberg X1: 535 p., 2010.

11. Eichfelder G., Jahn J. Foundations of Set-Semidefinite Optimization., In: Pardalos P., Rassias T., Khan A. (eds) Nonlinear Analysis and Variational Problems. Springer Optimization and Its Applications, vol 35. pp. 259-284. Springer, New York, NY, 2010.

12. Eichfelder G., Jahn J. Set-semidefinite optimization, Journal of Convex Analysis, 15, pp. 767-801, 2008. 
13. Glineur F. Conic optimization: an elegant framework for convex optimization, Belgian Journal of Operations Research, Statistics and Computer Science, 41, pp. 5?8,2001.

14. Kliemann L., Shirazi Sheykhdarabadi E., and Srivastav A. Price of anarchy for graph coloring games with concave payoff, JDG (by AIMS) 4(1), pp. 41-58, 2017.

15. Kostyukova O.I., Tchemisova T.V. Implicit optimality criterion for convex SIP problem with box constrained index set, TOP, 20 (2), pp. 475-502, 2012.

16. Kostyukova O.I., Tchemisova T.V. Optimality conditions for convex semi-infinite programming problems with finitely representable compact index sets, J. Optim. Theory Appl. 175(1), pp. 76-103, 2017.

17. Kostyukova O.I., Tchemisova T.V. Optimality conditions for linear copositive programming problems with isolated immobile indices, Optimization, vol. 69, issue 1, pp. 145-164, 2020.

18. Kostyukova O.I., Tchemisova T.V. Optimality criteria without constraint qualification for linear semidefinite problems, JMSJournal of Mathematical Sciences, 182 (2), pp. 126-143, 2012.

19. Kostyukova O.I., Tchemisova T.V. Sufficient optimality conditions for convex semi-infinite programming, Optimization Methods and Software, 25 (2), pp. 279-297, 2010.

20. Kostyukova O.I., Tchemisova T.V., Dudina O.S. Immobile indices and CQ-free optimality criteria for linear copositive programming problems, Set-Valued Var. Anal., 28, pp. 89-107, 2020.

21. Laraki, R., Lasserre, J.B. Semidefinite programming for min-max problems and games, Math. Program. 131, 305-332, 2012.

22. Motzkin, T. Copositive quadratic forms, National Bureau of Standards,Report 1818, pp. 11-12, 1952.

23. Özögür-Akyüz S., Akteke-Öztürk B., Tchemisova T., Weber G.-W. New optimization methods in Data Mining, In: Fleischmann B., Borgwardt KH., Klein R., Tuma A. (eds) Operations Research Proceedings. Springer, Berlin, Heidelberg, pp. 527-532, 2009.

24. Ramana, M. V., Tuncel, L., Wolkowicz H. Strong duality for semidefinite programming, SIAM J. Optimization, 7 (3), pp. 641-662, 1997.

25. Rockafellar R.T. Convex Analysis, Princeton Univ. Press, Vol.28, Princeton Univ. Pres, NJ, 470 p., 1970.

26. Shapiro A. Semi-infinite programming, duality, discretization and optimality conditions, Optimization, 58:2, pp. 133-161, 2009.

27. Sra S., Novozin S., and Wright S. J. Optimization for machine learning, Neural information processing series. Cambridge, MIT Press, 2012.

28. Stein O. How to solve a semi-infinite optimization problem, EJOR, vol. 223, Issue 2, pp. 312-320, 2012.

29. Stein O., Still G. On Optimality Conditions for Generalized Semi-Infinite Programming Problems, J. Optim. Theory Appl. 104, pp. 443-458, 2000

30. Tunçel L., Wolkowicz H. Strong duality and minimal representations for cone optimization, Comput. Optim. Appl. 53, pp. 619-648, 2013.

31. Vasant P. , Alparslan-Gök S.Z., and Weber G.-W. Handbook of Research on Emergent Applications of Optimization Algorithms, Volumes 1-2, IGI Global, Hershey PA, USA, 2017.

32. Weber G.-W., Kropat E., Alparslan Gök S.Z. Semi-Infinite and Conic Optimization in Modern Human Life and Financial Sciences under Uncertainty, In: ISI Proceedings of 20th Mini-EURO conference, pp. 180-185, 2008.

33. Wolkowicz H., Saigal R., Vandenberghe L. Eds. Handbook of semidefinite programming: theory, algorithms, and applications, Kluwer Academic Publishers, Boston, MA, 2000, 654 p.

34. Zaslavski A. Structure of approximate solutions of dynamic continuous time zero-sum games, JDG (by AIMS), 2014, 1(1): 153-179.

35. Zhang H., Bai Y., Fang C. Linear conic optimization models for robust credit risk optimization, Operations Research Transactions, v. $17(1), 86-97,2013$. 